$59.9 \pm 8.8$; male $19 \%$; spouse $17 \%$; parent $60 \%$ ). Qualitative content analysis revealed rich information about the characteristics of supportive support brokers, and also about support broker characteristics that were not supportive. Support brokers directly influenced many of the challenges caregivers identified. A responsive, imaginative, and involved support broker was identified as a major source of effective support. Conversely, frequent support broker turnover, limited knowledge and involvement, and the perception that the support broker was simply there to "check boxes" became sources of additional stress. Support brokers are uniquely positioned to help caregivers navigate the complexities of the healthcare system, thereby decreasing stress and strain. For example, many of the roadblocks that caregivers identified as difficult could often be negotiated by a support broker. Support brokers can serve as an avenue for caregivers to find out about additional supports within the PD program, as well as organizations outside of PD, such as disease-specific support groups, which caregivers identified as an additional source of support. This research is part of the Family Support Research \& Training Center.

\section{THE CHALLENGES OF SHARED DECISION-MAKING IN DEMENTIA CARE NETWORKS}

L. Groen - van de Ven ${ }^{1}$, C. Smits ${ }^{1}$, M. Span ${ }^{1}$, J.S. Jukema ${ }^{1}$, K. Coppoolse 2 , J. de Lange ${ }^{2}$, J. Eefsting ${ }^{3,4}$, M. VernooijDassen $^{5}$ 1. Research Group Innovating with Older Adults, Windesheim University of Applied Sciences, Zwolle, Netherlands, 2. Rotterdam University of Applied Sciences, Rotterdam, Netherlands, 3. Free University Medical Centre, Amsterdam, Netherlands, 4. Zonnehuisgroep IJssel-Vecht, Zwolle, Netherlands, 5. Radboud University Medical Centre, Nijmegen, Netherlands

Background

People with dementia want to be involved in the many decisions they have to make over time in order to adjust care to their values and preferences. Involving informal caregivers in decision-making can promote autonomy for people with dementia by using their extant capacities. However, informal caregivers have their own interests in the decisions made. Our aim was to describe the challenges of shared decisionmaking in dementia care networks.

Methods

Our study involves a multi-perspective qualitative study using face-to-face interviews with 113 respondents in 23 care networks consisting of 23 people with dementia, 44 of their informal caregivers, and 46 of their professional caregivers. The interview guide addressed the decision topics, the decision-making participants, and their contributions to the decision-making. We used content analysis to delineate categories and themes.

Results

The three themes that emerged describe the challenges of shared decision-making for dementia care networks: (1) adapting to a situation of diminishing independence, including shifting roles in the decision-making; (2) tensions in network interactions, resulting from different perspectives and interests and requiring agreement about what constitutes a problem in the situation; and (3) timing decisions well.

\section{Conclusion}

The challenges described have consequences for a shared decision-making approach in dementia care networks. Such an approach should (1) be flexible regarding the changing capabilities of the person with dementia to preserve his or her autonomy; (2) work towards a shared view about what constitutes a problem in the situation; and ( 3 ) be adjusted to the decision-making pace of the care network.

\section{INITIATIVE TO PROMOTE KNOWLEDGE OF GERIATRIC SYNDROMES IN MINORITY OLDER COMMUNITIES AND CAREGIVERS}

K. Alexander ${ }^{1,2}$, J. Nonaillada ${ }^{1}$, N. Gangai ${ }^{1}$, R. Costas Muniz ${ }^{1}$, B. Korc-Grodzicki ${ }^{1,2}, 1$. Geriatrics, Memorial Sloan Kettering Cancer Center, New York, New York, 2. Weill

Cornell Medical College, New York, New York

In addition to the ageing of the population, a significant sociodemographic change in the United States is the growth in the number of minorities. Minorities and older adults are particularly vulnerable to receiving suboptimal healthcare. The Health Resources and Services Administration (HRSA), is the primary Federal agency for improving and achieving health equity. Queens County in New York City is the most ethnically diverse urban area in the world. Funded by a grant from HRSA, in collaboration with various local community organizations, the Geriatric Resource Interprofessional Program (GRIP) at Memorial Sloan Kettering Cancer Center (MSKCC) spearheaded a multi-prong educational initiative on geriatric syndromes targeting minority communities in Queens County. To date, 13 sessions were conducted at 7 community centers in Queens. A total of 521 people who spoke 13 different primary languages attended. The mean age of the participants was 63 (27-99, median 67); 53\% were women, $8 \%$ were born in the USA, $56 \%$ were born in Bangladesh, $22 \%$ in India. Consecutive interpretation of the lectures was performed to the predominant language of the group; written materials were translated. Pre and post test questionnaires were administered to measure understanding. A focus group was set up to identify the needs of caregivers of older adults, and educational sessions have been offered based on those needs at main Community Centers. Main challenges included differences in literacy, multiple cultures and languages. Successes and barriers faced in implementing the educational initiative, as well as pre and post-test results will be presented.

\section{CULTIVATING FAMILY CAREGIVER RESILIENCE AND PREVENTING AVOIDABLE HOSPITALIZATIONS IN DEMENTIA}

T. Sadak ${ }^{2}$, E. Ishado ${ }^{1}$, B. Gaster ${ }^{3}$, L.N. Gitlin ${ }^{4}$, K.C. Buckwalter ${ }^{5}$, S. Borson ${ }^{3,1}, 1$. University of Washington School of Nursing, Mountlake Terrace, Washington, 2. university of Washington School of Nursing, Seattle, Washington, 3. University of Washington School of Medicine, Seattle, Washington, 4. John Hopkins University School of Nursing, Baltimore, Maryland, 5. University of Iova School of Nursing, Iowa, Iowa

Enhancing and maintaining family caregiver (CG) selfcare is a national priority for long-term societal wellbeing. The National Alzheimer's Plan Act recognizes widespread 